\title{
Online Spectrum Allocation for Cognitive Cellular Network Supporting Scalable Demands
}

\author{
Jianfei Wang * $\dagger$ \\ jfwang213@gmail.com
}

\author{
Jinzhao $\mathrm{Su}^{* \dagger}$ \\ sujinzhao@gmail.com \\ *State Key Laboratory of Virtual Reality and Systems \\ ${ }^{\dagger}$ School of Computer Science of BeiHang University(BUAA)
}

\author{
Wei $\mathrm{Wu}^{* \dagger}$ \\ wuwei@buaa.edu.cn
}

\begin{abstract}
We advocate an online spectrum allocation system for cognitive cellular network supporting scalable demands. Our system's demands processing model is online model, which means that allocation result is returned immediately. To solve the tough issues of online spectrum allocation, we try to balance the spectrum utilization and future coming demands. A state machine is introduced to allocate spectrum efficiently, and at different state system have different procedures to provide service to customers. To make good use of scalable property of demand and to increase fairness among customers, the concept of satisfaction degree is introduced. We also analyze our system's state and its transition theoretically and give the derivation of every parameter of system and the method to calculate them. Enough work is done to evaluate the system with real data evaluation and results show that the system is efficient and robust under different cases in which the customers' arrival rate and service time are varied.
\end{abstract}

\section{INTRODUCTION}

With the rapid development of mobile communication system, the limited radio spectrum is becoming crowded. So some researchers are trying their best to improve the mobile communication system with the concept of Dynamic Spectrum Access (DSA) networks[2], which aims to break the spectrum access barrier and enable their end-users dynamically access spectrum, and we can call this class of cellular network cognitive cellular network. Cognitive cellular network can make use of spectrum which primary users don't occupy to provide service for customers. In this paper, we focus on the coordinated cognitive cellular networks where customers have to get the permission of the base station before communication.

Buddhikot introduce the cognitive radio network into cellular network and try to improve mobile communication. His paper [2] gives some important spectrum access concepts of scope, access fairness, stickness and spectrum utilization. Sachs propose a cognitive cellular architecture within the TV Spectrum in paper [8]. The approaches to primary-secondary spectrum sharing is overlay operation. In their approach, to compensate for the interference caused by overlay transmissions, the cognitive cellular base station cooperates with the TV system.

In paper [2], Buddhikot divided the demand processing model into two classes, one is batched and the other is online. In batched model, user proposes a request and after some interval system start to deal with all users' request. In contrast, in online model, when user proposes a request, system will allocation some spectrum to user immediately. There are much work of batched model, such as [3]. Recently there are also some work on online demand processing model. In paper [11], authors propose some strategies to achieve truthful spectrum auction for cognitive radio network. The demand processing model in that paper is online model.

With the development of methods of spectrum access,such as OFDMA, and software defined radio, the bandwidth and modulation of communication can be adaptive to environment. At the same time, the transmission rate of scalable demand can be varied. Most of our requirements are browsing web pages, downloading files, watching video, listening to music and so on. As we know, browsing web pages and downloading file are scalable demands because if the network is not very good, you can fulfill your demand with more time. Techniques like Scalable Video Coding (SVC) enable the scalable property of watching video[9]. There are also some audio coding which enable the scalable property [10].

To make good use of the demand's scalable property, we introduce the concept of satisfaction degree denoted by $r$. If a user comes up with a request $w_{a}$ and the system's current satisfaction is $r_{t}$, the user will get spectrum with bandwidth $w_{g}=w_{a} * r_{t} . w_{a}$ is the max spectrum bandwidth required by the application and if customers get spectrum with bandwidth more than $w_{a}$, some spectrum will be wasted. So $r$ must follow the restrain of $r \leq 1$.

The rest of paper is organized as follows: We introduce our system model in section II. Section III does some theoretical analysis to the model where we give the method to calculate the parameters of model. In section IV, we evaluate our system with real wireless data. Finally, Section V summarizes our conclusions.

\section{SYSTEM MODEL}

\section{A. Spectrum Model and Spectrum Access Method}

Because we focus on spectrum allocation of one base station, the spectrum model is simple. Because all users of a base station are communicating with the base station, the channels of different users must be different. The conflict graph of our customers is complete graph $K_{n}$. The spectrum of adjacent base station should be different, because the interference range is larger than communication range. The spectrum allocation 
among adjacent base station is out of the scope of this paper. In this paper we discuss the spectrum allocation among users of the same base station. We assume the base station holds spectrum whose bandwidth is $w_{0}$ and spectrum of adjacent base station is different.

\section{B. The System Assumption}

For practicality and simplicity, we make some assumptions on the system. We assume system will deal with demands of different applications individually. With the same application, we assume the maximum demands of different users are the same. This assumption is rational for browsing webpages because the webpages are almost the same. In [4], the authors take a research about the video of YouTube and the results show that the bit rate of videos are almost the same in website of YouTube. The max demands of application is denoted by $w_{a}$.

When modeling cellular network system, we take four factors into count: Arrival of user's request,Customer's service time,Queueing rules and The capacity of the system. For simplicity and fairness, we assume the queuing rules are First Come First Served(FCFS). The sequence of user's request is important to the system. We assume the process of customer's arrival is a poisson process.

$$
P\{X(s+t)-X(s)=k\}=e^{-\lambda t} \frac{(\lambda t)^{k}}{k !}
$$

In (1) the $\lambda$ is average arrival rate. The expect of the number of user arriving in interval $\mathrm{t}$ is $\lambda * t$. And the interarrival time distribution $\mathrm{A}(\mathrm{x})$ is a exponential distribution. We can also get the expect of interarrival time $\mathrm{x}$ is:

$$
E(x)=\frac{1}{\lambda}
$$

The user's service time is also important to the system. We assume the time distribution of user's service time is a exponential distribution and the density of service time $b(t)$ is :

$$
b(t)=\left\{\begin{array}{cc}
\mu e^{-\mu t}, & t \geq 0 \\
0, & t<0
\end{array}\right.
$$

With (3), we can get the expect of service time is :

$$
E(t)=\frac{1}{\mu}
$$

From (4), we can get the average departure rate $\frac{1}{E(t)}$ is $\mu$.

The input of the system is the arriving customers. We denote the inputs by $\alpha(t)$ where

$$
\alpha(t) \triangleq \text { number of arrivals in }(0, t)
$$

The output of the system is the customers who have ended his service. We denote the outputs by $\beta(t)$ where

$$
\beta(t) \triangleq \text { number of departures in }(0, t)
$$

With the customer's arrival process and the customer's departure process, we can get the number of active customers at time instance $\mathrm{t}$ by $N(t)$

$$
N(t)=\alpha(t)-\beta(t)
$$

The system has a max capacity $N_{0}$ which is the max number of active customers. If the satisfaction of some application is too low, the user's request can not be fulfilled. For example, in video on demand system, the lowest level of video's bitrate is more than zero and the minimum of satisfaction exist. We denote the minimum of satisfaction by $r_{\min }$. We can get such equation:

$$
N_{0} \leq \frac{w_{0}}{r_{\min } * w_{a}}
$$

\section{State Machine of System}

To balance the spectrum utilization and future demands, we involve state machine into our system. At different state and different time, satisfaction degree of system will be different. The state of online spectrum allocation system can be divided into four classes:

a. Rich State. This state shows that the spectrum of the system is in low efficiency. The base station can improve the satisfaction of customers.

b. Normal State. This state indicates that system is normal. The satisfaction of customers is fit for the system.

c. Poor State. In this state the free spectrum of the system is not enough. The base station should decrease the satisfaction of coming customers in order to fulfill more customers' request.

d. Terrible State. This state shows that the free spectrum of system is exhausted. The system has to reject customer's request.

We can distinguish the states from each other by percentage of free spectrum. We define four value $f_{1}, f_{2}, f_{3}$ which is percentage of occupied spectrum and no more than 1 . The state assignment of state machine is:

$$
\text { State }=\left\{\begin{array}{cc}
S_{\text {rich }}, & f \leq f_{1} \\
S_{\text {normal }}, & f_{1} \leq f \leq f_{2} \\
S_{\text {poor }}, & f_{2} \leq f \leq f_{3} \\
S_{\text {terrible }}, & f_{3} \leq f \leq 1
\end{array}\right.
$$

\section{Transition of State}

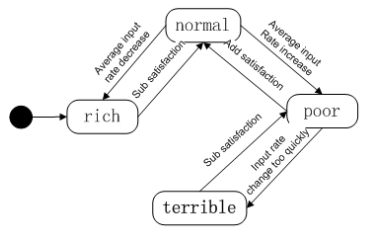

Fig. 1. State Machine

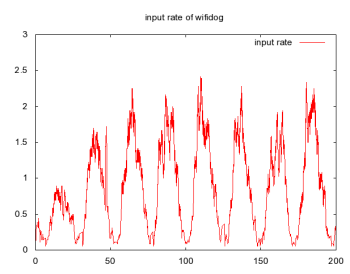

Fig. 2. estimated input rate
Fig 1 shows the transition of state machine. System can adjust the satisfaction to drive the transfer of State Machine. If system is at the Normal State, it is showed that the satisfaction, which is denoted by $r_{2}$, is fit for the system. We denote corresponding arrival rate and departure rate by $\lambda_{2}$ and $\mu_{2}$. If system is at the Poor State, we can infer that the satisfaction is too high and should be reduced to $r_{3}$. Before reducing satisfaction degree, system has try to know about 
environment now. System tries to estimate arrival rate and departure rate which are denoted by $\lambda_{3}$ and $\mu_{3}$. If system is at the Rich State, it is inferred that the satisfaction is too low and can be improved to $r_{1}$. At the same time system also will estimate arrival rate $\lambda_{1}$ and departure rate $\mu_{1}$.

\section{THEORETICAL ANALYZE}

To derive the important property of the system and learn system's how to respond to the changes of environment, we do some theoretical analysis firstly.

\section{A. Stabilization of Normal State}

The system can be thought of as an alternated of $\mathrm{M} / \mathrm{M} / N_{0} / N_{0}$ queueing system. In [5], the probability of that the number of active customer is $\mathrm{k}$ is:

$$
\begin{gathered}
p_{k}=\left\{\begin{array}{cc}
p_{0} \frac{\lambda^{k}}{k ! \mu^{k}} & 0 \leq k \leq N_{0} \\
0 & k>N_{0}
\end{array}\right. \\
p_{0}=\frac{1}{1+\sum_{k=1}^{N_{0}} \frac{\lambda^{k}}{k ! \mu^{k}}}
\end{gathered}
$$

The desired state of system is Normal State in which system can balance spectrum utilization and future demand. In this state, the spectrum efficiency is high and meanwhile it can also resist some changes. The spectrum occupied can be denoted by $w_{\text {occupy }}$. System try to stay at the normal state by adjusting the satisfaction to $r_{2}$. We can derive that:

$$
E\left(w_{\text {occupy }}\right)=w_{0} * f_{2}, \quad r_{t}=r_{2}
$$

Referred to equilibrium solution, we can assume that the satisfaction of all active users is $r_{2}$ because of long time at Normal State. We can get:

$$
E\left(w_{\text {occupy }}\right)=E(N(t)) * w_{a} * r_{2}
$$

From (10), we can derive $E(N(t))$ as:

$$
E(N(t))=\sum_{i=0}^{N_{0}} i * p_{i}=p_{0}+\frac{\lambda}{\mu} \sum_{i=0}^{N_{0}-1} i * p_{i} \approx \frac{\lambda}{\mu}
$$

By substituting (14) and (13) into (12), we can get:

$$
r_{2}=\frac{f_{2} * w_{0}}{w_{a}} * \frac{\mu_{2}}{\lambda_{2}}
$$

From equation (15), we can get that $r_{2}$ is related to average arrival rate $\lambda_{2}$ and average departure rate $\mu_{2}$. To derive precise value of $r_{2}$, we have to think of a method to estimate $\lambda_{2}$ and $\mu_{2}$ which will be introduced later.

\section{B. Transition of System}

When system is in the state of Rich State or Poor State, it is implied that the satisfaction is not fit for the arrival rate and departure rate. When system is in Rich State, system should improve the user satisfaction to $r_{1}$. From (15) and temporal average arrival rate $\mu_{1}$ and departure rate $\lambda_{1}$,we can get:

$$
r_{1}=\frac{f_{2} * w_{0}}{w_{a}} * \frac{\mu_{1}}{\lambda_{1}}
$$

The transition from Poor State to Normal State is similar, we can get:

$$
r_{3}=\frac{f_{2} * w_{o}}{w_{a}} * \frac{\mu_{3}}{\lambda_{3}}
$$

\section{How to Define State Boundary}

The state Boundaries $f_{1}, f_{2}, f_{3}$ are very important to system. We need to refer to history data to define state boundaries.

Before giving the method of calculating boundaries, we infer that the user's arrival rate is periodic within one day. In paper [6], authors use the method of principal component analysis to analyze the network's throughout and they find that the throughout is periodic. We also do an experiment with data from CRAWDAD[7] to collect the customer's arrival rate. Fig 2 show the results of our experiment.

Based on history data, we can get $P(\lambda, t)$ whose parameters are arrival average rate and time instance. When we evaluate system, the input rate and time instance can be discretized. $\lambda$ is one of $\left(\lambda_{i}(1 \leq i \leq N)\right)$, where $\mathrm{N}$ is the number of $\lambda_{i}$ after discretization.

1) The Boundary between Normal and Poor State: The occupied spectrum ratio $f_{3}$ is the boundary between Normal and Poor State. If the system occupied spectrum ratio is more than $f_{3}$, system is at Poor state and system will decrease the user satisfaction. If the ratio is less than $f_{3}$, system will be at the Normal State and satisfaction degree will keep stable.

Because of different arrival rate at different time instance of one day, the boundary between Normal State and Poor State $f_{3}$ is different. And there is only small difference between arrival rates of the same time of different days, $f_{3}$ at one time instance of one day can be the same as $f_{3}$ at the same time instance of another day. This is to say we can calculate $f_{3}$ based on the average arrival rate and service time. We denote occupied spectrum ratio by $S r$.

The input rate $\lambda_{n}$ correspond to the average arrival rate. We denote it by:

$$
\lambda_{n}=\sum_{i=1}^{N} \lambda_{i} * P\left(\lambda_{i}, t\right)
$$

Corresponding satisfaction $r_{n}$ can be calculated by equation (15). If the value of $r_{n}$ is more than 1 , we can infer at this time of one day the arrival rate is too small and system can not reach the state of Poor State. In this case, the value of $f_{3}$ is not critical and we can simply set $f_{3}=\frac{f_{2}+1}{2}$. Now we focus on the case when the value of $r_{n}$ is less than 1 . The event of $S r=f_{3}$ is equal to the event of $N(t)=k_{n}$, where $k_{n}=\frac{f_{3} * w_{o}}{w_{a} * r_{n}}$.

We define $f_{3}$ as the occupied spectrum ratio that the probability of reaching is too small, such as $1 \%$, without drastic change on input rate. This is to say that:

$$
P\left(N(t)>=k_{n} \mid \lambda_{t}=\lambda_{n}\right)<1 \%
$$

Substitute (10) into (19), we can get:

$$
\sum_{i=k_{n}}^{N_{0}} p_{i}=p_{0} * \sum_{i=k_{n}}^{N_{0}} \frac{\lambda^{i}}{i ! \mu^{i}}<0.01
$$


From (20), we calculate the value of $k_{n} . f_{3}$ can be calculated with equation:

$$
f_{3}=\frac{r_{n} * w_{a} * k_{n}}{w_{0}}
$$

2) The boundary between Normal and Rich State: The boundary between Normal and Rich State is $f_{1}$. When defining $f_{1}$, we have to balance the users' satisfaction and the stability. When $f_{1}$ is high, the probability of false alarm about rich spectrum will be high and the stability of system is low. When $f_{1}$ is low, much spectrum will be wasted and users' satisfaction will be decreased. As same as $f_{3}, f_{1}$ is different at different time instance of one day and the same at the same time instance of different day. $f_{1}$ is also calculated based on the averaged arrival rate. The arrival rate $\lambda_{n}$ denotes the average arrival rate calculated by (18). Correspond satisfaction $r_{n}$ can be calculated by (15). Similar to definition of $f_{3}$, the method of calculating $f_{1}$ is :

$$
P\left(N(t)<=k_{m} \mid \lambda_{t}=\lambda_{n}\right)<1 \% .
$$

From (22), we can get the value of $k_{m} . f_{1}$ can be calculated with equation:

$$
f_{1}=\frac{r_{n} * k_{m} * w_{a}}{w_{0}}
$$

3) Middle Occupied Frequency Ratio of Normal State: The most important parameter of system is the middle frequency of Normal State. Because at most of time system should be at the state of Normal state, system frequency utility mostly depends on the occupied frequency ratio, which is $f_{2}$. From this aspect, we hope $f_{2}$ is larger and more consumers can be served.

From (20), we can get that at any time instance t, $f_{3}$ is depended on $k_{n}$. Similar to $f_{3}$, there is a value $k_{2}$ correspond to $f_{2}$. The equation is $f_{2}=\frac{k_{2} * w_{a} * r_{2}}{w_{0}}$. So we can get:

$$
f_{3}(t)=m(t) * f_{2}(t)
$$

From the subsection of calculating the value of $r_{3}$, we get that as a result of the variation of arrival rate and service time, when $f_{3}$ is closer to 1 , the probability of blocking will be higher. From this aspect, we hope $f_{2}$ is smaller and the probability of blocking will be small. If blocking is common, consumers will complain and choose another service provider.

So service provider can get the value of $f_{2}$ by maximizing the number of consumers.

\section{Estimation of the Arrival Rate and Service Time}

Online property make it important to estimate the arrival rate and service time. The autocorrelation of real data give us some inspiration about the method of estimation.

In Fig 3 which is based on the wifidog data [7], we collect the number of arrival customers in one hour and the average service time in one hour and calculate the autocorrelation. From the left graph of Fig 3, we can get that the correlation among the arrival customers in different hours is very big. So we can get estimation of average arrival rate with the model of seasonal ARMA model. In the right figure of Fig
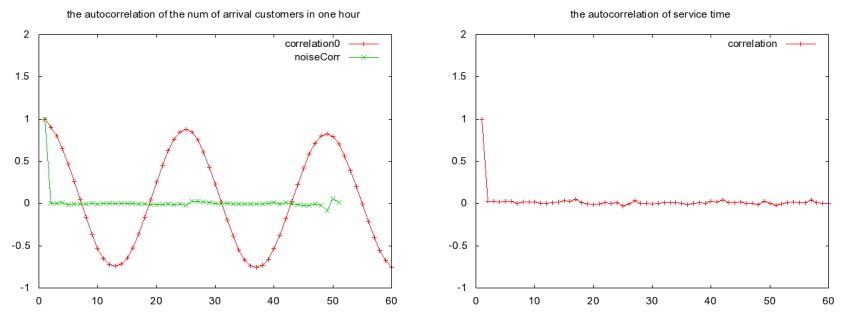

Fig. 3. autocorrelation of arrival rate and service time

3 , the correlation of service time is so little that we estimate the average service time with maximum likelihood method of estimating the average of exponential distribution.

1) Estimation of Arrival Rate: From the left part of Fig 3, we can infer the arrival rate can be described by an seasonal ARMA model. If we collect the arrival customers in one hour, we can get the cycle of arrival rate is 24 . So the seasonal arma model of arrival rate can be denoted by

$$
\phi(B) \tilde{z}(t)=\phi^{\prime}(B)\left(1-B^{24}\right) \tilde{z}(t)=\theta(B) a(t)
$$

where $\mathrm{B}$ is an operator and $B * a(t)=a(t-1)$. In [1],Box gives the method of deriving $\varphi(B)$ and $\theta(B)$ with autocorrelation and partial autocorrelation.

2) Estimation of Departure Rate: We use maximum likelihood method to estimate the value of departure rate $\mu$. We denote estimated value by $\hat{\mu}$. We can get that:

$$
\hat{\mu}=\frac{N_{\text {est }}}{\sum_{i=-N_{\text {est }}}^{-1} t_{i}}
$$

where $t_{i}$ is the last ith customer's service time. $N_{e s t}$ is the number of recent requested service time. When estimating departure rate there are also active customers in the system. Equation (26) does not take active customers into count and can be more exact. Because of memoryless property of exponential distribution, expect of active customers' left service time are the same as expect of service time and is $\frac{1}{\mu}$. We can get that:

$$
\frac{1}{\hat{\mu}}=\frac{\sum_{i=-N_{e s t}}^{-1} t_{i}+T_{\text {active }}+\frac{1}{\mu} * N(t)}{N_{\text {est }}+N(t)}
$$

where $T_{\text {active }}$ is elapsed service time of active customers before estimating. In(27), we can replace $\mu$ with $\hat{\mu}$. After transformation of (27), we get:

$$
\hat{\mu}=\frac{N_{\text {est }}}{\sum_{i=-N_{\text {est }}}^{-1} t_{i}+T_{\text {active }}}
$$

\section{Evaluation}

In this section, we will evaluate our model with real data which is from CRAWDAD, a community resource for archiving wireless data at Dartmouth. The data is collected by a non-profit organization and is measured by recording users' 

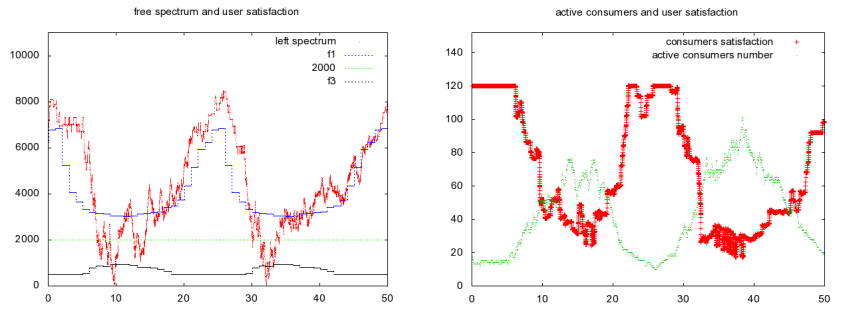

Fig. 4. Evaluation with Real Data

session data on wifi hotspot. It counts 45,000 users over 140 hotspots. Though it is not a cellular network, it is significant to learn user's behavior. Because of the differences between wifi network and cellular network, we ignore the incoming data and the outgoing data transferred and use the login time and logout time data.

We divide the data into two part, one for training the parameters and the other for evaluation the system.

3) Training the parameters of model: As a result of popular video service, we assume users' request $w_{a}$ is $300 \mathrm{~Hz}$ and middle frequency occupied ratio is $80 \%$. After analysis of training data. The largest input rate of one day is 2.5 customer per second. The average service time 4320s. We assume system's frequency denoted by $w_{0}$ is $10 \mathrm{Mhz}$.

To calculate the value of $f_{3}$ and $f_{2}$, we discretize the time domain. We record average input rate every hour and at last get the average input rate of one hour. The values of $f_{3}$ and $f_{1}$ are showed in the left part of Fig 4.

With the real data [7] and the method proposed in [1], we can derive the parameters of $\phi(B)$ and $\theta(B)$. We can check the correction in the way of calculating the correlation of $a(t)$. In the left part of Fig 3, the line with label noiseCorr is the result of correlation of $a(t)$. The correlation of $a(t)$ is very small. From this we can infer that the arma model is fit for estimating the value of arrival rate.

4) Evaluation the System: Fig 2 show the customer's arrival rate, we can get that the arrival rate changes very severely, from 0.03 to 2.5 . The average service time is $4320 \mathrm{~s}$. The data is very challenging to our system because average service time is so long that system is not very dynamic. Fig 4 is the results of real data evaluation. The left graph is about the free spectrum and the right graph is the consumers' satisfaction degree and active consumers number in system. In left graph, the $\mathrm{x}$-axis is time and our evaluation lasts for two days. The y-axis is free spectrum in hz. At the beginning, as a result of small amount of consumers, satisfaction degree and free spectrum of system is high. When it is eight o'clock system goes into Normal State and satisfaction degree start to decrease. After that system stay at the Normal State. When it is ten o'clock, because of increased arrival rate, system goes into the Poor State. After decreasing consumers' satisfaction degree, system goes back to Normal State. When it is at the midnight, because of severely declined arrival rate, though system increases satisfaction degree, system goes to Rich State.

From the results, we can conclude that in the environment of Wi-Fi, our system's online spectrum allocation algorithm is well. Familiar with Wi-Fi, cellular network is centralized and consumers are communicating with central node. After this evaluation with real data, we can infer that in cellular network our system performs well.

\section{CONCLUSION}

In this paper, we propose a system for online spectrum allocation for scalable demands in cognitive cellular network. To handle the issues produced by online property, we come up with a method of state machine to balance the spectrum utilization and future demands. We introduce a concept of users' satisfaction degree to make good use of scalable property of demand. With theoretical analysis of system, we give the method of calculating parameters of system. At last we use collections of real wireless data to evaluate our system. The results prove that in cellular network our system will perform well.

\section{ACKNOWLEDGMENT}

We have to thank the anonymous reviewers for the helpful suggestions. We need to thank contributors of CRAWDAD for their open data. The authors would like to thank Education Ministry of China for their support of this work under the National Foundation of the Doctoral Program (200800060018), the support of Aviation Science Foundation (2009ZD51038) and also the support of Beijing Natural Science Foundation(4102032).

\section{REFERENCES}

[1] G.E.P. Box and G.M. Jenkins. Time series analysis: forecasting and control. Prentice Hall PTR Upper Saddle River, NJ, USA, 1994.

[2] M. Buddhikot and K. Ryan. Spectrum management in coordinated dynamic spectrum access based cellular networks. In Proc. of IEEE DySPAN, pages 299-307, 2005.

[3] L. Cao, L. Yang, X. Zhou, Z. Zhang, and H. Zheng. Optimus: SINRDriven Spectrum Distribution via Constraint Transformation. In New Frontiers in Dynamic Spectrum, 2010 IEEE Symposium on, pages 1-12. IEEE, 2010.

[4] X. Cheng, C. Dale, and J. Liu. Statistics and social network of youtube videos. In Quality of Service, 2008. IWQoS 2008. 16th International Workshop on, pages 229-238. IEEE, 2008.

[5] L. Kleinrock. Queueing systems, volume 1: theory, 1975

[6] A. Lakhina, K. Papagiannaki, M. Crovella, C. Diot, E.D. Kolaczyk, and N. Taft. Structural analysis of network traffic flows. ACM SIGMETRICS Performance evaluation review, 32(1):61-72, 2004.

[7] Michael Lenczner, Benoit Grgoire, and Fran?ois Proulx. CRAWDAD trace set ilesansfil/wifidog/session (v. 2007-08-27). Downloaded from http://crawdad.cs.dartmouth.edu/ilesansfil/wifidog/session, August 2007.

[8] J. Sachs, I. Maric, and A. Goldsmith. Cognitive Cellular Systems within the TV Spectrum. In New Frontiers in Dynamic Spectrum, 2010 IEEE Symposium on, pages 1-12. IEEE, 2010.

[9] T. Schierl, T. Stockhammer, and T. Wiegand. Mobile video transmission using scalable video coding. IEEE Transactions on Circuits and Systems for Video Technology, 17(9):1204-1217, 2007.

[10] J. Sjoberg, M. Westerlund, and A. Lakaniemi. Q. Xie," RTP Payload Format and File Storage Format for the Adaptive Multi-Rate (AMR) and Adaptive Multi-Rate Wideband (AMR-WB) Audio Codecs. Technical report, RFC 4867, April 2007.

[11] S. Sodagari, A. Attar, and S.G. Bilen. Strategies to Achieve Truthful Spectrum Auctions for Cognitive Radio Networks Based on Mechanism Design. In New Frontiers in Dynamic Spectrum, 2010 IEEE Symposium on, pages 1-6. IEEE, 2010. 